VoL. 47 (1993) [457-464]

\title{
A DECISION PROBLEM FOR VARIETIES OF COMMUTATIVE SEMIGROUPS
}

\author{
W.L. CAO
}

For a first order formula $P: \forall x_{1} \ldots \forall x_{n} \exists y_{1} \ldots \exists y_{m}\left(u\left(x_{1}, \ldots, x_{n}, y_{1}, \ldots, y_{m}\right) \equiv\right.$ $\left.v\left(x_{1}, \ldots, x_{n}, y_{1}, \ldots, y_{m}\right)\right)$, where $u$ and $v$ are two words on the alphabet $\left\{x_{1}, \ldots, x_{n}, y_{1}, \ldots, y_{m}\right\}$, and a finite set $E$ of semigroup identities with $x y \equiv y x$ in it, we prove that it is decidable whether $P$ follows from $E$, that is whether all the semigroups in the variety defined by $E$ satisfy $P$.

\section{INTRODUCTION}

One of the fundamental decision problems in algebra is the so-called word problem: decide whether a identity $P$ follows from a set $E$ of identities. It has been proved that the word problems for semigroups and groups are both undecidable, but decidable for commutative semigroups and commutative groups [1].

Some important semigroup properties cannot be described by semigroup identities, for example, $S$ is
(i) regular;
(ii) simple;
(iii) a group, et cetera.

But they can be described as $S$ satisfies

(i) $\forall x \exists y(x y x \equiv x)$;

(ii) $\forall x \forall y \exists u \exists v(u x v \equiv y)$;

(iii) $\forall x \forall y \exists z(x z \equiv y) \wedge \forall x \forall y \exists z(z x \equiv y)$, et cetera.

In this paper, we consider the following decision problem for semigroups: decide whether $P$ follows from a set $E$ of semigroup identities, where $P$ is the first order formula

$$
\forall x_{1} \ldots \forall x_{n} \exists y_{1} \ldots \exists y_{m}\left(u\left(x_{1}, \ldots, x_{n}, y_{1}, \ldots, y_{m}\right) \equiv v\left(x_{1}, \ldots, x_{n}, y_{1}, \ldots, y_{m}\right)\right)
$$

and $u, v$ are two words on the alphabet $\left\{x_{1}, \ldots, x_{n}, y_{1}, \ldots, y_{m}\right\}$. Notice $P$ will reduce to an identity if the existential variables $y_{j}$ do not appear in $P$.

Received 28 May 1992

Copyright Clearance Centre, Inc. Serial-fee code: 0004-9729/93 SA2.00+0.00. 
We say a semigroup $S$ satisfies $P$ if for any $s_{1}, \ldots, s_{n}$ in $S$, there are $w_{1}, \ldots, w_{m}$ in $S$, such that

$$
u\left(s_{1}, \ldots, s_{n}, w_{1}, \ldots, w_{m}\right)=v\left(s_{1}, \ldots, s_{n}, w_{1}, \ldots, w_{m}\right)
$$

holds in $S$.

We say $P$ follows from $E$, written as $E \Vdash P$, if whenever a semigroup $S$ satisfies every identity in $E, S$ satisfies $P$ also, that is all semigroups in the variety $[E]$ defined by $E$ satisfy $P$.

For any set $\Sigma$, let $\Sigma^{+}$and $\Sigma^{*}$ denote, respectively, the free semigroup and free monoid generated by $\Sigma$. For a word $w \in \Sigma^{*}$ and a letter $a \in \Sigma,\left(\begin{array}{l}w \\ a\end{array}\right)$ denotes the number of times the letter $a$ appears in $w$.

We regard a semigroup identity $u \equiv v$ as a pair of words $(u, v) \in V^{+} \times V^{+}$, where $V$ is a countably infinite set of variables.

Let $A=\left\{a_{1}, a_{2}, \ldots, a_{n}, \ldots\right\}$, and $A_{n}=\left\{a_{1}, a_{2}, \ldots, a_{n}\right\}, n \geqslant 1$. We define the following binary relations on $A_{n}^{+}$:

(i) $w \rho_{n}^{E} w^{\prime}$, if for some homomorphism $\varphi: V^{+} \rightarrow A_{n}^{+}$, and $u \equiv v$ in $E$, $\varphi(u)=w, \varphi(v)=w^{\prime}$;

(ii) $w \underset{E}{\leftrightarrow} w^{\prime}$, if for some $u, v, x, y \in A_{n}^{*}, w=x u y, w^{\prime}=x v y$, and $u \rho_{n}^{E} v$ or $v \rho_{n}^{E} u$

(iii) $w \underset{E}{\stackrel{*}{\longrightarrow}} w^{\prime}$, if $w=w^{\prime}$ or for some $z_{1}, z_{2}, \ldots, z_{k} \in A_{n}^{+}, w=z_{1}, w^{\prime}=z_{k}$, $z_{i} \overleftrightarrow{E}^{z_{i+1}}, i \leqslant k-1$

So $\underset{E}{\stackrel{*}{\longrightarrow}}$ is the congruence generated by $\rho_{n}^{E}$, and $A_{n}^{+} / \underset{E}{\stackrel{*}{\longrightarrow}}$ is the relntively free semigroup in $[E]$ over the set $\left\{\left[a_{i}\right] \mid 1 \leqslant i \leqslant n\right\}$, where $\left[a_{i}\right]=\left\{w \in A_{n}^{+} \mid a_{i} \underset{E}{\stackrel{*}{\longrightarrow}} w\right\} . P$ follows from $E$ if and only if there are $w_{1}, w_{2}, \ldots, w_{m} \in A_{n}^{+}$, such that

$$
u\left(a_{1}, \ldots, a_{n}, w_{1}, \ldots, w_{m}\right) \stackrel{*}{\stackrel{*}{\leftarrow}} v\left(a_{1}, \ldots, a_{n}, w_{1}, \ldots, w_{m}\right) .
$$

\section{The RESULTS}

From now on, we assume that $x y \equiv y x$ is in $E$, that is $[E]$ is a variety of commutative semigroups.

Let $E^{\prime}=\left\{u \equiv v \in E \mid\left(\begin{array}{l}u \\ x\end{array}\right) \neq\left(\begin{array}{l}v \\ x\end{array}\right)\right.$ for some $\left.x \in V\right\}$. Without loss of generality, we assume

$$
\begin{aligned}
E & =E^{\prime} \cup\{x y \equiv y x\}, \\
E^{\prime} & =\left\{x_{1}^{p_{i 1}} x_{2}^{p_{i 2}} \ldots x_{k}^{p_{i k}} \equiv x_{1}^{p_{i 1}^{\prime}} x_{2}^{p_{i 2}^{\prime}} \ldots x_{k}^{p_{i k}^{\prime}} \mid i=1,2, \ldots, L\right\}, \quad\left(\text { if } E^{\prime} \neq \emptyset\right. \text { ) }
\end{aligned}
$$




$$
\begin{aligned}
& u\left(x_{1}, \ldots, x_{n}, y_{1}, \ldots, y_{m}\right)=x_{1}^{s_{1}} \ldots x_{n}^{s_{n}} y_{1}^{t_{1}} \ldots y_{m}^{t_{m}} \\
& v\left(x_{1}, \ldots, x_{n}, y_{1}, \ldots, y_{m}\right)=x_{1}^{s_{1}^{\prime}} \ldots x_{n}^{s_{n}^{\prime}} y_{1}^{t_{1}^{\prime}} \ldots y_{m}^{t_{m}^{\prime}} .
\end{aligned}
$$

Lemмa 1. If $E^{\prime}=0$, then it is decidable whether $E \Vdash P$.

Proof: $E \Vdash P$ if and only if there are $w_{j}=\prod_{i=1}^{n} a_{i}^{z_{i j}} \in A_{n}^{+}, j \leqslant m$, such that $u\left(a_{1}, \ldots, a_{n}, w_{1}, \ldots, w_{m}\right) \stackrel{*}{\rightleftarrows} v\left(a_{1}, \ldots, a_{n}, w_{1}, \ldots, w_{m}\right)$. But $E^{\prime}=\emptyset$ implies that for any words $w, w^{\prime} \in A_{n}^{+}, w \underset{E}{\stackrel{*}{\rightleftarrows}} w^{\prime}$ if and only if $\left(\begin{array}{l}w \\ a_{i}\end{array}\right)=\left(\begin{array}{l}w^{\prime} \\ a_{i}\end{array}\right)$, for all $a_{i} \in A_{n}$. So $E \Vdash P$ if and only if the following linear system with integer unknowns $x_{i j}$ is solvable:

$$
\left\{\begin{array}{l}
\sum_{j=1}^{m} T_{j} x_{i j}=S_{i}, \quad i=1,2, \ldots, n ; \\
\sum_{i=1}^{n} x_{i j}>0, \quad j=1,2, \ldots, m ; \\
x_{i j} \geqslant 0, \quad i=1,2, \ldots, n, j=1,2, \ldots, m^{\prime}
\end{array}\right.
$$

where $T_{j}=t_{j}-t_{j}^{\prime}, S_{i}=s_{i}^{\prime}=s_{i}$. Without loss of generality, we assume that all $T_{j} \neq 0$ (because we can delete $y_{j}$ from $P$, if $T_{j}=0$ ).

Case (i). There are some $T_{\alpha}, T_{\beta}, 1 \leqslant \alpha, \beta \leqslant m, T_{\alpha}>0, T_{\beta}<0$. In this case $\left(^{*}\right)$ is solvable if and only if $\left(T_{1}, T_{2}, \ldots, T_{m}\right) \mid S_{i}, 1 \leqslant i \leqslant n$. Clearly, (*) is solvable implies $\left(T_{1}, T_{2}, \ldots, T_{m}\right) \mid S_{i}, 1 \leqslant i \leqslant n$. On the other hand, if $\left(T_{1}, T_{2}, \ldots, T_{m}\right) \mid S_{i}$, then there are integers $\alpha_{i j}, \sum T_{j} \alpha_{i j}=S_{i}$. Let us assume that $T_{1}>0, T_{m}<0$. Take $\beta_{i j}=-T_{m} N_{i j}+\alpha_{i j}, 1 \leqslant j \leqslant m-1, \gamma_{i}=\alpha_{i m}+\sum N_{i j} T_{j}$. We have

$$
\sum_{j<m} T_{j} \beta_{i j}+T_{m} \gamma_{i}=S_{i},
$$

and if $N_{i j}$ are sufficiently large (in particular, $N_{i i}$ ), we have $\beta_{i j}>0, \sum_{j<m} T_{j} \beta_{i j}>S_{i}$, then we have $\gamma_{i}=\left(S_{i}-\sum_{j<m} T_{j} \beta_{i j}\right) / T_{m}>0$.

Case (ii). $T_{j}>0, j=1,2, \ldots, m$. (The case of $T_{j}<0$ can be treated similarily.) In this case, any $S_{i}<0$ implies $\left({ }^{*}\right)$ is unsolvable. If $S_{i} \geqslant 0$ for all $i=1,2, \ldots, n$, any solution of $\left({ }^{*}\right)$ will satisfy $0 \leqslant x_{i j} \leqslant S_{i}$, so it is decidable whether or not $\left(^{*}\right)$ has solutions.

From now on, we assume that $E^{\prime} \neq 0$. Define

$$
Q_{E}=\min \left\{q>0 \mid\left(a_{1}^{q}, a_{1}^{p+q}\right) \text { or }\left(a_{1}^{p+q}, a_{1}^{q}\right) \in \rho_{1}^{E} \text { for some } p>0\right\},
$$

and

$$
D_{E}=\left(g_{11}, \ldots, g_{1 k}, g_{21}, \ldots, g_{2 k}, \ldots, g_{L 1}, \ldots, g_{L k}\right),
$$

that is $D_{E}$ is the greatest common divisor of $g_{i j}$, where $g_{i j}=p_{i j}-p_{i j}^{\prime}, 1 \leqslant i \leqslant L$, $1 \leqslant j \leqslant k$. 
Lemma 2. For any $w, w^{\prime} \in A_{n}^{+}, w \underset{E}{\stackrel{*}{*}} w^{\prime} \operatorname{implies}\left(\begin{array}{l}w \\ a_{i}\end{array}\right)-\left(\begin{array}{c}w^{\prime} \\ a_{i}\end{array}\right)=0 \bmod D_{E}$, $1 \leqslant i \leqslant n$.

Proof: It is enough to prove that $\left(\begin{array}{c}w \\ a_{i}\end{array}\right)-\left(\begin{array}{l}w \\ a_{i}\end{array}\right)=0 \bmod D_{E}, 1 \leqslant i \leqslant n$, for all $\left(w, w^{\prime}\right) \in \rho_{n}^{E}$. Let $f \equiv g \in E^{\prime}, f=x_{1}^{p_{01}} \ldots x_{k}^{p_{0 k}}, g=x_{1}^{p_{11}^{\prime}} \ldots x_{k}^{p_{d k}^{\prime}}$. Then $\varphi: V^{+} \rightarrow A_{n}^{+}$ is a homomorphism, $\varphi(f)=w, \varphi(g)=w^{\prime}$, and $\left(\begin{array}{c}\varphi\left(x_{j}\right) \\ a_{i}\end{array}\right)=\alpha_{i j}, j=1,2, \ldots, k$. We have

$$
\left(\begin{array}{c}
w \\
a_{i}
\end{array}\right)-\left(\begin{array}{c}
w^{\prime} \\
a_{i}
\end{array}\right)=\sum_{j=1}^{k}\left(p_{d j}-p_{a j}^{\prime}\right) \alpha_{i j}=0 \quad\left(\bmod D_{E}\right) .
$$

LEMMA 3. $E \Vdash x^{Q_{E}} \equiv x^{Q_{E}+D_{E}}$.

Proof: Let $D_{E}=\sum \beta_{i j} g_{i j}$ and $M=\max \left\{1,1-\beta_{i j}(1 \leqslant i \leqslant L, 1 \leqslant j \leqslant k)\right\}$. We have

$$
D_{E}=\sum\left(M+\beta_{i j}\right) g_{i j}-\sum M g_{i j} .
$$

Because $M \geqslant 1, M+\beta_{i j} \geqslant 1$, for any sufficiently large number $N$,

$$
a_{1}^{N} \underset{E}{\stackrel{*}{\rightleftarrows}} a_{1}^{N+D_{E}},
$$

because we can find an implication chain corresponding to equation $\left({ }^{*}\right)$. Assume $\left(a_{1}^{Q_{E}}, a_{1}^{Q_{E}+p}\right) \in \rho_{n}^{E}$, we have

$$
a_{1}^{Q_{E}} \underset{E}{\stackrel{*}{\rightleftarrows}} a_{1}^{Q_{E}+N_{P}} \underset{E}{\stackrel{*}{\rightleftarrows}} a_{1}^{Q_{E}+N_{P}+D_{E}} \underset{E}{\stackrel{*}{\rightleftarrows}} a_{1}^{Q_{E}+D_{E}} .
$$

For convenience, we write $w_{1} \Leftrightarrow w_{2}$ if for some $\left(w, w^{\prime}\right)$ or $\left(w^{\prime}, w\right) \in \rho_{n}^{E},\left(\begin{array}{c}w_{1} \\ a_{i}\end{array}\right)-$ $\left(\begin{array}{l}w \\ a_{i}\end{array}\right)=\left(\begin{array}{c}w_{2} \\ a_{i}\end{array}\right)-\left(\begin{array}{c}w^{\prime} \\ a_{i}\end{array}\right), i=1,2, \ldots, n$, and $\left(\begin{array}{c}w_{1} \\ a_{i}\end{array}\right) \neq\left(\begin{array}{c}w_{2} \\ a_{i}\end{array}\right)$ for some $a_{i} \in A_{n}$. Note $w_{1} \leftrightarrow w_{2}$ implies $w_{1} \stackrel{*}{\stackrel{*}{\rightleftarrows}} w_{2}$. On the other hand, $w_{1} \underset{E}{\stackrel{*}{\rightleftarrows}} w_{2}$ implies either

(i) $\left(\begin{array}{c}w_{1} \\ a_{i}\end{array}\right)=\left(\begin{array}{c}w_{2} \\ a_{i}\end{array}\right), 1 \leqslant i \leqslant n$; or

(ii) for some $z_{1}, z_{2}, \ldots, z_{s} \in A_{n}^{+}, w_{1}=z_{1}, w_{2}=z_{s}, z_{i} \Leftrightarrow z_{i+1}, i \leqslant s-1$.

Now, let $N=\max \left\{k, Q_{E}\right\}$. For a word $a_{1}^{k_{1}} a_{2}^{k_{2}} \ldots a_{n}^{k_{n}} \in A_{n}^{+}$, construct sets $B_{i}(w)$, $C_{i}(w)$, and functions $F_{i}^{w}: A_{n} \rightarrow\{0,1\}$ iteratively.

(i) Construct

$$
\begin{aligned}
B_{0}(w) & =\{w\}=\left\{a_{1}^{k_{1}} a_{2}^{k_{2}} \ldots a_{n}^{k_{n}}\right\} . \\
F_{0}^{w}\left(a_{j}\right) & = \begin{cases}1, & \text { if } k_{j} \geqslant N \\
0, & \text { otherwise; }\end{cases} \\
C_{0}(w) & =\left\{a_{1}^{p_{1}} a_{2}^{p_{2}} \ldots a_{n}^{p_{n}} \mid p_{j}=\min \left\{k_{j}, N\right\}, j \leqslant n\right\} .
\end{aligned}
$$


(ii) For any $i>0$, construct

$$
\begin{aligned}
& B_{1}(w)=\left\{a_{1}^{m_{1}} a_{2}^{m_{2}} \ldots a_{n}^{m_{n}} \mid a_{1}^{m_{1}} a_{2}^{m_{2}} \ldots a_{n}^{m_{n}} \Leftrightarrow w^{\prime},\right. \text { for some } \\
& \left.w^{\prime} \in C_{i-1}(w)\right\} \text {, } \\
& F_{1}^{w}\left(a_{j}\right)= \begin{cases}1, & \text { if } F_{i-1}^{w}\left(a_{j}\right)=1 \text { or } m_{j} \geqslant N \text { for some } \\
& a_{1}^{m_{1}} a_{2}^{m_{2}} \ldots a_{n}^{m_{n}} \in B_{1}(w) \\
0, & \text { otherwise; }\end{cases} \\
& C_{i}(w)=\left\{a_{1}^{p_{1}} a_{2}^{p_{2}} \ldots a_{n}^{p_{n}} \mid \text { for some } a_{1}^{m_{1}} a_{2}^{m_{2}} \ldots a_{n}^{m_{n}} \in B_{1}(w)\right. \text {, } \\
& \left.p_{j}=\min \left\{N, m_{j}\right\}, j \leqslant n\right\} \\
& \cup\left\{a_{1}^{p_{1}} a_{2}^{p_{2}} \ldots a_{n}^{p_{n}} \mid \text { for some } a_{1}^{m_{1}} a_{2}^{m_{2}} \ldots a_{n}^{m_{n}} \in B_{i}(w)\right. \text {, } \\
& \left.p_{j}=F_{i}^{w}\left(a_{j}\right) N+\left(1-F_{i}^{w}\left(a_{j}\right)\right) m_{j}\right\} \\
& \cup C_{i-1}(w) \text {. }
\end{aligned}
$$

LEMмA 4. The following statements are true:

(i) $\quad C_{i}(w) \subseteq C_{i+1}(w), i \geqslant 0$;

(ii) $C_{i}(w) \subseteq\left\{a_{1}^{m_{1}} a_{2}^{m_{2}} \ldots a_{n}^{m_{n}} \mid m_{j} \leqslant N, j=1,2, \ldots, n\right\}$;

(iii) $C_{i}(w)=C_{i+1}(w)$ implies $C_{i}(w)=C_{i+p}(w)$ and $F_{i+1}^{w}=F_{i+p}^{w}$, for any $p>0$.

Proof: Self-evident.

Let $i$ be the number such that $C_{i}(w)=C_{i+1}(w)$, define

$$
\begin{aligned}
F^{w}= & F_{i+1}^{w} \\
C(w)= & \left\{a_{1}^{m_{1}} a_{2}^{m_{2}} \ldots a_{n}^{m_{n}} \mid \text { for some } a_{1}^{q_{1}} a_{2}^{q_{2}} \ldots a_{n}^{q_{n}} \in C_{i}(w),\right. \\
& \left.m_{j}=F^{w}\left(a_{j}\right) N+\left(1-F^{w}\left(a_{j}\right)\right) q_{j}\right\} .
\end{aligned}
$$

\section{LEMMA 5. For any $i \geqslant 0$}

(i) $F_{i}^{w}\left(a_{j}\right)=1$ implies $w \stackrel{*}{\stackrel{*}{\longrightarrow}} w a_{j}^{D_{E}}$;

(ii) for any $a_{1}^{p_{1}} a_{2}^{p_{2}} \ldots a_{n}^{p_{n}} \in C_{i}(w)$, there is $a_{1}^{q_{1}} a_{2}^{q_{2}} \ldots a_{n}^{q_{n}} \in A_{n}^{+}$, where $q_{k}=$ $p_{k}$ if $F_{i}^{w}\left(a_{k}\right)=0$, otherwise $q_{k} \geqslant N, k=1,2, \ldots, n$, and $w \stackrel{*}{\rightleftarrows}$ $a_{1}^{q_{1}} a_{2}^{q_{2}} \ldots a_{n}^{q_{n}}$.

Proof: We prove this lemma by induction. For $i=0$, it is clearly true. Now assume it is true for $i-1$. If $F_{i}^{w}\left(a_{j}\right)=1$ and $F_{i-1}^{w}\left(a_{j}\right)=1$, then $w \stackrel{*}{\rightleftarrows} w a_{j}^{D_{E}}$, by inductive assumption. If $F_{i}^{w}\left(a_{j}\right)=1$ but $F_{i-1}^{w}\left(a_{j}\right)=0$, then there are $a_{1}^{m_{1}} a_{2}^{m_{2}} \ldots a_{n}^{m_{n}} \in$ $B_{i}(w), a_{1}^{h_{1}} a_{2}^{h_{2}} \ldots a_{n}^{h_{n}} \in C_{i-1}(w), a_{1}^{m_{1}} a_{w}^{m_{2}} \ldots a_{n}^{m_{n}} \Longleftrightarrow a_{1}^{h_{1}} a_{2}^{h_{2}} \ldots a_{n}^{h_{n}}, m_{j} \geqslant N$. By 
inductive assumption, there are $a_{1}^{f_{1}} a_{2}^{f_{2}} \ldots a_{n}^{f_{n}} \in A_{n}^{+}, a_{1}^{f_{1}} a_{2}^{f_{2}} \ldots a_{n}^{f_{n}} \underset{E}{\stackrel{*}{\rightleftarrows}} w, f_{j}=h_{j}$, but $f_{k} \geqslant h_{k}$ for all $k \neq j$. Now we have

$$
\begin{aligned}
& w \underset{E}{\stackrel{*}{\rightleftarrows}} a_{1}^{f_{1}} a_{2}^{f_{2}} \ldots a_{j}^{h_{j}} \ldots a_{n}^{f_{n}} \\
& \stackrel{*}{\stackrel{*}{\longrightarrow}} a_{1}^{m_{1}^{\prime}} a_{2}^{m_{2}^{\prime}} \ldots a_{j}^{m_{j}} \ldots a_{n}^{m_{n}^{\prime}} \quad \text { (where } m_{k}^{\prime}=f_{k}-h_{k}+m_{k} \text { ) } \\
& \stackrel{*}{\longrightarrow} a_{1}^{m_{1}^{\prime}} a_{2}^{m_{2}^{\prime}} \ldots a_{j}^{m_{j}+D_{E}} \ldots a_{n}^{m_{n}^{\prime}} \quad \text { (by Lemma 3) } \\
& \stackrel{*}{\stackrel{*}{\longrightarrow}}\left(a_{1}^{m_{1}^{\prime}} a_{2}^{m_{2}^{\prime}} \ldots a_{j}^{m_{j}} \ldots a_{n}^{m_{n}^{\prime}}\right) a_{j}^{D_{E}} \\
& \stackrel{*}{\stackrel{H}{\longrightarrow}} w a_{j}^{D_{E}} \text {. }
\end{aligned}
$$

Now take $a_{1}^{p_{1}} \ldots a_{n}^{p_{n}} \in C_{i}(w)$ if $a_{1}^{p_{1}} \ldots a_{n}^{p_{n}} \in C_{i-1}(w)$, then by the inductive assumption and since $w \underset{E}{\stackrel{*}{\rightleftarrows}} w a_{j}^{D_{E}}$ for all $j$ with $F_{i}^{w}\left(a_{j}\right)$, we know there is $a_{1}^{q_{1}} \ldots a_{n}^{q_{n}} \in A_{n}^{+}$, where $q_{k}=p_{k}$ if $F_{i}^{w}\left(a_{k}\right)=0$, and $q_{k} \geqslant N$, if $F_{i}^{w}\left(a_{k}\right)=0, k=1,2, \ldots, n$, and $\boldsymbol{w} \stackrel{*}{\rightleftarrows}$ $a_{1}^{q_{1}} \ldots a_{n}^{q_{n}}$. If $a_{1}^{p_{1}} \ldots a_{n}^{p_{n}} \notin C_{i-1}(w)$, let $a_{1}^{m_{1}} \ldots a_{n}^{m_{n}} \in B_{i}(w), a_{1}^{h_{1}} \ldots a_{n}^{h_{n}} \in C_{i-1}(w)$, $a_{1}^{f_{1}} \ldots a_{n}^{f_{n}} \in A_{n}^{+}$, such that

(i) $p_{j}=m_{j}$, if $F_{i}^{w}\left(a_{j}\right)=0$;

(ii) $a_{1}^{h_{1}} \ldots a_{n}^{h_{n}} \Longleftrightarrow a_{1}^{m_{1}} \ldots a_{n}^{m_{n}}$

(iii) $x \underset{E}{\stackrel{*}{\rightleftarrows}} a_{1}^{f_{1}} \ldots a_{n}^{f_{n}}$, and $f_{j} \geqslant N$ if $F_{i-1}^{w}\left(a_{j}\right)=1$, otherwise $f_{j}=h_{j}$, $j=1,2, \ldots, n$.

Then we have

$$
\begin{aligned}
w \underset{E}{\stackrel{*}{\rightleftarrows}} a_{1}^{g_{1}} \ldots a_{n}^{g_{n}} & \text { (by (iii)) } \\
\stackrel{*}{\stackrel{*}{\rightleftarrows}} a_{1}^{m_{1}^{\prime}} \ldots a_{n}^{m_{n}^{\prime}}, & \text { (by (ii)) }
\end{aligned}
$$

where $m_{j}^{\prime}=m_{j}+\left(g_{j}-h_{j}\right)$. Because $w \underset{E}{\stackrel{*}{\rightleftarrows}} w a_{j}^{D_{E}}$ where $F_{i}^{w}\left(a_{j}\right)=1$, letting $M>$ $N / D_{E}$, we have

$$
w \stackrel{*}{\stackrel{*}{\rightleftarrows}} a_{1}^{q_{1}} \ldots a_{n}^{q_{n}},
$$

where $q_{j}=m_{j}^{\prime}+M D_{E}>N$, if $F_{i}^{w}\left(a_{j}\right)=1$, otherwise $q_{j}=m_{j}^{\prime}=m_{j}=p_{j}$.

Leмma 6. Let $w=a_{1}^{p_{1}} \ldots a_{n}^{p_{n}}$. If there is $w^{\prime}=a_{1}^{q_{1}} \ldots a_{n}^{q_{n}} \in A_{n}^{+}$, such that $w \underset{E}{\stackrel{*}{\longrightarrow}} w^{\prime}$ and $q_{j} \geqslant N$, then $F^{w}\left(a_{j}\right)=1$.

Proof: If $p_{j} \geqslant N$, then $F^{w}\left(a_{j}\right)=F_{0}^{w}\left(a_{j}\right)=1$. Now assume $p_{j}<N$. If $F^{w}\left(a_{j}\right)=0$, let

$$
w=z_{0} \Longleftrightarrow z_{1} \Longleftrightarrow \ldots \Longleftrightarrow z_{t-1} \Longleftrightarrow z_{t} \Longleftrightarrow \ldots \Longleftrightarrow z_{\imath}=w^{\prime},
$$


where $z_{i}=a_{1}^{q_{i 1}} \ldots a_{n}^{q_{i n}}, i \leqslant s$, and $q_{i j}<N$ for all $i \leqslant t-1$, but $q_{i j} \geqslant N$. There are $z_{i}^{\prime} \in C_{i}(w), z_{i}^{\prime}=a_{1}^{p_{i 1}} \ldots a_{n}^{p_{i n}}$, where $p_{i j}=q_{i j}$ if $F_{i}^{w}\left(a_{j}\right)=0$, otherwise $p_{i j}=N$, $i \leqslant t-1$. Imitate $z_{t-1} \Longleftrightarrow z_{t}$, we have $z_{t-1}^{\prime} \Longleftrightarrow a_{1}^{h_{1}} \ldots a_{n}^{h_{n}}$, where $h_{j}=q_{t j} \geqslant N$. So $F^{w}\left(a_{j}\right)=1$.

LEMMA 7. Let $w=a_{1}^{m_{1}} \ldots a_{n}^{m_{n}}, w^{\prime}=a_{1}^{q_{1}} \ldots a_{n}^{q_{n}}$. If $w \underset{E}{\stackrel{*}{\rightleftarrows}} w^{\prime}$, then there is $a_{1}^{p_{1}} \ldots a_{n}^{p_{n}} \in C(w)$, where $p_{j}=q_{j}$ if $F^{w}\left(a_{j}\right)=0$, otherwise $p_{j}=N$.

Proof: Let

$$
w=z_{0} \Longleftrightarrow z_{1} \Longleftrightarrow \ldots \Longleftrightarrow z_{t}=w^{\prime},
$$

where $z_{i}=a_{1}^{q_{i 1}} \ldots a_{n}^{q_{n}^{i n}}, i \leqslant t$. Just as we did in the proof of Lemma 6 , take the same $z_{i}^{\prime} \in C_{i}(w)$. So there is $a_{1}^{p_{1}} \ldots a_{n}^{p_{n}} \in C(w)$, where $p_{j}=N$ if $F^{w}\left(a_{j}\right)=1$, otherwise $p_{j}=q_{i j}=q_{j}$.

LEMMA 8. Let $w=a_{1}^{m_{1}} \ldots a_{n}^{m_{n}}, w^{\prime}=a_{1}^{q_{1}} \ldots a_{n}^{q_{n}}$. Then $w \underset{E}{\stackrel{*}{\rightleftarrows}} w^{\prime}$, if and only if

(i) $m_{i}-q_{i}=0\left(\bmod D_{E}\right), i \leqslant n$;

(ii) $F^{w}=F^{w^{\prime}}$;

(iii) $C(w) \cap C\left(w^{\prime}\right) \neq \emptyset$.

Proof: Clearly, $w \underset{E}{\stackrel{*}{\rightleftarrows}} w^{\prime}$ implies the conditions (i), (ii), and (iii), by Lemma 2, $5,6,7$. Now assume that (i), (ii) and (iii) are true. For convenience, we assume that $F^{w}\left(a_{j}\right)=1$ for all $j \leqslant d, F^{w}\left(a_{j}\right)=0$ for all $j>d$, where $d \geqslant 0$. (ii) and (iii) imply that there are $w_{1}=a_{1}^{f_{1}} \ldots a_{d}^{f_{d}} a_{d+1}^{p_{d+1}} \ldots a_{n}^{p_{n}}, w_{2}=a_{1}^{h_{1}} \ldots a_{d}^{h_{d}} a_{d+1}^{p_{d+1}} \ldots a_{n}^{p_{n}} \in A_{n}^{+}, f_{j}$, $h_{j} \geqslant N$, and $w \underset{E}{\stackrel{*}{\rightleftarrows}} w_{1}, w^{\prime} \underset{E}{\stackrel{*}{\rightleftarrows}} w_{w}$. For any $j \leqslant d, f_{j}-h_{j}=\left(f_{j}-m_{j}\right)+\left(m_{j}-q_{j}\right)+$ $\left(a_{j}-h_{j}\right)=0\left(\bmod D_{E}\right)$, by (i) and Lemma 2 , and therefore $\underset{j}{f_{j}} \stackrel{*}{\rightleftarrows} a_{j}^{h_{j}}$, by Lemma 3. Hence $w \underset{E}{\stackrel{*}{\rightleftarrows}} w_{1} \underset{E}{\stackrel{*}{\rightleftarrows}} w_{2} \underset{E}{\stackrel{*}{\longrightarrow}} w^{\prime}$.

Now we state and prove the main result of this paper.

Theorem 9. Let $E$ and $P$ be as described before, $E^{\prime} \neq 0$. It is decidable whether $E \Vdash P$.

PROOF: $w=a_{1}^{s_{1}} \ldots a_{n}^{s_{n}}, w^{\prime}=a_{1}^{s_{1}^{\prime}} \ldots a_{n}^{s_{n}^{\prime}}$. We can assume $F^{w^{\prime}}\left(a_{j}\right)=1$ if and only if $j \leqslant p$, for some $p \geqslant 0$. We need to consider three cases.

(i) All $t_{j}$ and $t_{j}^{\prime}=0$. In this case, $P$ reduces to an identity, and $E \Vdash P$ if and only if $w \underset{E}{\stackrel{*}{*}} w^{\prime}$. By Lemma 8, this is decidable.

(ii) Some $t_{j}^{\prime}>0$, and some $t_{j}>0$. In this case, $E \Vdash P$ if and only if $\left(D_{E}, T_{1}, T_{2}, \ldots, T_{m}\right) \mid S_{i}, i=1,2, \ldots, n$, where $T_{j}=t_{j}-t_{j}^{\prime}, S_{i}=s_{i}-s_{i}$. Be- 
casue there are $w_{1}, \ldots, w_{m} \in A_{n}^{+}$, such that

$$
u\left(a_{1}, \ldots, a_{n}, w_{1}, \ldots, w_{m}\right) \stackrel{\leftrightarrow}{\stackrel{*}{\longrightarrow}} v\left(a_{1}, \ldots, a_{n}, w_{1}, \ldots, w_{m}\right),
$$

the following system with integer unknowns $x_{i j}$ is solvable:

$$
S_{i}+\sum T_{j} x_{i j}=0\left(\bmod D_{E}\right), \quad i=1,2, \ldots, n ;
$$

and this in turn implies $\left(D_{E}, T_{1}, T_{2}, \ldots, T_{m}\right) \mid S_{i}, i=1,2, \ldots, n$. On the other hand, $\left(D_{E}, T_{1}, T_{2}, \ldots, T_{m}\right) \mid S_{i}$ means $\left(^{* *}\right)$ is solvable, and if $x_{i j}, i \leqslant n, j \leqslant m$, is a solution, then $M D_{E}+x_{i j}, i \leqslant n, j \leqslant m$, is also a solution, for any $M>0$. Therefore, there exist $w_{1}, \ldots, w_{m} \in A_{n}^{+}$, such that

$$
\begin{aligned}
& \left(\begin{array}{c}
u \\
a_{j}
\end{array}\right)>N,\left(\begin{array}{c}
v \\
a_{j}
\end{array}\right)>N, \\
& \left(\begin{array}{c}
u \\
a_{j}
\end{array}\right)-\left(\begin{array}{c}
v \\
a_{j}
\end{array}\right)=0\left(\bmod D_{E}\right), \\
& j=1,2, \ldots, n ;
\end{aligned}
$$

where $u=u\left(a_{1}, \ldots, a_{n}, w_{1}, \ldots, w_{m}\right), v=v\left(a_{1}, \ldots, a_{n}, w_{1}, \ldots, w_{m}\right)$. Let

$$
X=a_{1}^{h_{1}} \ldots a_{n}^{h_{n}}, \quad Y=a_{1}^{f_{1}} \ldots a_{n}^{f_{n}},
$$

where $h_{j}=\left(\begin{array}{c}u \\ a_{j}\end{array}\right), f_{j}=\left(\begin{array}{c}v \\ a_{j}\end{array}\right), j \leqslant n$. We have

$$
u \stackrel{*}{\stackrel{*}{\longrightarrow}} X \underset{E}{\stackrel{*}{\longrightarrow}} Y \stackrel{*}{\stackrel{*}{\rightleftarrows}} v .
$$

(iii) All $t_{j}^{\prime}=0$, but some $t_{j}>0$. We can assume that all $t_{j}>0$. In this case, it is not difficult to see that $E \Vdash P$ if and only if

(a) $F^{w} \leqslant F^{w^{\prime}}$ (that is $F^{w}\left(a_{j}\right)=0$ for all $j>p$ );

(b) $\left(t_{1}, t_{2}, \ldots, t_{m}, D_{E}\right) \mid s-s^{\prime}, i \leqslant p$;

(c) for some $a_{1}^{f_{1}} \ldots a_{n}^{f_{n}} \in C\left(w^{\prime}\right)$, there exist $x_{i j} \geqslant 0$, such that $s_{i}+\sum_{j=1}^{m} t_{j} x_{i j}=$ $f_{i}, i \geqslant P+1$;

so it is also decidable.

\section{References}

[1] B. Benninghofen, S. Kemmerich and M.M. Richter, Systems of reductions (Springer-Verlag, Berlin, Heidelberg, New York, 1987). 